\title{
MHC associations of ankylosing spondylitis in East Asians are complex and involve non-HLA-B27 HLA contributions
}

Geng Wang ${ }^{1,2 \dagger}$, Tae-Hwan Kim ${ }^{3 \dagger}$, Zhixiu Li ${ }^{4 \dagger}$, Adrian Cortes ${ }^{5}$, Kwangwoo Kim6 ${ }^{6}$ So-Young Bang ${ }^{3}$, Paul Leo ${ }^{4}$, Matthew A. Brown ${ }^{7 *}$ and Huji Xu, $u^{1,8,9^{*}}$

\begin{abstract}
Background: The association of HLA-B*27 with AS is amongst the strongest of any known association of a common variant with any human disease. Nonetheless, there is strong evidence indicating that other HLA-B alleles are involved in the disease. European ethnicity studies have demonstrated risk associations with $H L A-B^{*} 40$ and multiple other HLA-B, HLA-A, and HLA class II alleles, and demonstrated that in that ethnic group, the amino acid sequence at position 97 in HLA-B is the key determinant of HLA associations with AS. A recent study in Korean AS cases and controls additionally identified association at HLA-C*15:02. In the current study, we examined the MHC associations of AS in an expanded East Asian cohort.
\end{abstract}

Methods: A total of 1637 Chinese, Taiwanese, and Korean AS cases meeting the modified New York Criteria for AS, and 1589 ethnically matched controls, were genotyped with the Illumina Immunochip, including a dense coverage of the MHC region. HLA genotypes and amino acid composition were imputed using the SNP2HLA programme using the Han-MHC reference panel based on the data of Han Chinese subjects $(n=9689)$, and association tested using logistic regression controlling for population stratification effects.

Results: A strong association was seen with $H L A-B^{*} 27$ (odds ratio $(O R)=205.3, P=5.76 \times 10^{-244}$ ). Controlling for this association, the strongest risk association is seen with $\mathrm{HLA}-\mathrm{C}^{*} 15$ at genome-wide significant level $(\mathrm{OR}=7.62, P=9.30 \times$ $\left.10^{-19}\right)$, and confirmed association is also seen with HLA-B*40 at suggestive level $\left(\mathrm{OR}=1.65, P=2.54 \times 10^{-4}\right)$. At amino acid level, the strongest association seen in uncontrolled analysis was with histidine at position 114 in HLA-B $\left(P=7.24 \times 10^{-241}\right)$, but conditional analyses suggest that the primary amino acid associations are with lysine at position 70 and asparagine at position 97. Restriction of the ERAP1 association with HLA-B27-positive AS, previously reported in European subjects, was confirmed in East Asians.

\footnotetext{
*Correspondence: matt.brown@kcl.ac.uk; huji_xu@tsinghua.edu.cn

${ }^{\dagger}$ Geng Wang, Tae-Hwan Kim, and Zhixiu Li contributed equally to this work.

'Guy's \& St Thomas' NHS Foundation Trust and King's College London NIHR Biomedical Research Centre, London, England

'Department of Rheumatology and Immunology, Shanghai Changzheng Hospital, Second Military Medical University, Shanghai, China

Full list of author information is available at the end of the article
}

C C The Author(s). 2020 Open Access This article is licensed under a Creative Commons Attribution 4.0 International License, which permits use, sharing, adaptation, distribution and reproduction in any medium or format, as long as you give appropriate credit to the original author(s) and the source, provide a link to the Creative Commons licence, and indicate if changes were made. The images or other third party material in this article are included in the article's Creative Commons licence, unless indicated otherwise in a credit line to the material. If material is not included in the article's Creative Commons licence and your intended use is not permitted by statutory regulation or exceeds the permitted use, you will need to obtain permission directly from the copyright holder. To view a copy of this licence, visit http://creativecommons.org/licenses/by/4.0/ The Creative Commons Public Domain Dedication waiver (http://creativecommons.org/publicdomain/zero/1.0/) applies to the data made available in this article, unless otherwise stated in a credit line to the data. 
(Continued from previous page)

Conclusions: This study confirms in East Asians that the HLA associations of AS are multiple, including previously reported associations at $H L A-B^{*} 27, H L A-B^{*} 40$, and $H L A-C^{*} 15$, as well as novel association with HLA-DQB $1^{*} 04$. The HLA-B associations are driven by the amino acids at positions 70 and 97, in the B pocket of $H L A-B$.

Keywords: Ankylosing spondylitis, HLA, Association

\section{Background}

Ankylosing spondylitis (AS) is a highly heritable rheumatic disease characteristically causing chronic inflammation of the spine and sacroiliac joints, as well as in some patients affecting the peripheral joints, the anterior uvea, and less commonly other organs. The worldwide distribution of AS is closely related to the prevalence of $H L A$ $B * 27$, although the underlying mechanism remains unclear. Whilst the $H L A-B * 27$ allele is found in approximately $85 \%$ of patients, there is strong evidence indicating that other $H L A-B$ alleles and $M H C$ genes are involved in the disease, as well as non-MHC loci.

Direct genotyping studies in European case-control cohorts have demonstrated risk associations consistently with $H L A-B * 40$ and variably reported associations with multiple other HLA-B, HLA-A, and HLA class II alleles. The development of accurate HLA imputation methods from single nucleotide polymorphism (SNP) microarray data has enabled far larger case-control studies to be performed, with, for the first time, proper control for population stratification effects. Using this approach and studying 22,647 AS cases and controls of European descent, Cortes et al. demonstrated that the amino acid sequence of HLA-B at position 97, in the epitope-binding groove, is the key determinant of HLA associations with AS. After controlling for the associated alleles in $H L A-B$, independent associations with variants in the $H L A-A$, $H L A-D P B 1$, and $H L A-D R B 1$ loci were observed [1].

Differences in $H L A-B * 27$ subtype distributions between Asian and European descent populations have been well reported, and further non-HLA-B*27 HLA class I associations in East Asian AS have been reported. Also using HLA imputation methods, a study in $654 \mathrm{Ko-}$ rean cases of AS and 3166 controls additionally identified association at $H L A-C^{*} 15: 02$ [2]. Additionally, using direct genotyping in 360 Han Chinese AS cases and 350 controls with no genomic control for population stratification, risk association of $H L A-B * 40$ and protective association of $H L A-B * 07$ have been demonstrated [3].

In this study, using HLA imputation methods, we analyse the associations of AS with major histocompatibility complex (MHC) polymorphisms to identify functional and potentially causal variants using a large cohort of East Asian ancestry AS cases and controls [4]. In addition to our primary analysis of this cohort, we perform fine mapping of the $\mathrm{MHC}$ region with imputation of SNPs, HLA class I and II classical alleles, and amino acid residues within the classical HLA proteins. In addition to $H L A-B * 27$, we identify further HLA-B and other HLA class I and II alleles associated with AS.

\section{Methods}

\section{Subjects and SNP data}

A total of 1637 Chinese, Taiwanese, and Korean AS cases meeting the modified New York Criteria for AS [5] as confirmed by qualified rheumatologists, and 1589 ethnically matched controls (Table 1 ), were genotyped with the customised SNP array (Illumina Immunochip [6]), including a dense coverage of the MHC region. Cohort descriptions and genotyping protocols are as previously reported [4]. By standard quality control procedures, SNPs with a minor allele frequency of at least $1 \%$ (MAF $>0.01$ ), call rates of $\geq 0.98$, and $P$ values in HardyWeinberg disequilibrium tests $\leq 10^{-7}$ were analysed in this study. To confirm ethnicity, we performed a continental principal components analysis (PCA), merging the study genotype data available from 51 available populations genotyped by Illumina 650Y from the Human Genome Diversity Panel (HGDP-CEPH) [7]. Cases or controls lying more than 6 standard deviations from the population mean on principal components (PCs) 1-10 were then excluded.

\section{HLA imputation and association analysis}

We conducted a 2-step imputation. We densely imputed SNPs across the MHC using the Michigan Imputation Server [8] and the 1000 Genomes Phase 3 reference dataset (26 populations across the world), then further using the Han-MHC reference panel [9], to ensure maximum SNP coverage to enable accurate imputation of HLA-B alleles, including of particular interest, HLA-B27. Using this SNP data and the Han Chinese reference panel $(N=9869)$, the programme SNP2HLA was used to impute the classic HLA alleles and amino acid residues

Table 1 Demographic summary of the study cohort

\begin{tabular}{lllll}
\hline & Cases & Controls & Total & Percentage \\
\hline Chinese & 764 & 683 & 1447 & 45 \\
Taiwanese & 214 & 179 & 393 & 12 \\
Korean & 659 & 727 & 1386 & 43 \\
Total & 1637 & 1589 & 3226 & - \\
\hline
\end{tabular}


of the 8 HLA genes (HLA-A, HLA-B, HLA-C, HLA$D P B 1, \quad H L A-D Q B 1, \quad H L A-D R B 1, \quad H L A-D P A 1, \quad H L A-$ $D Q A 1)$ in a total of 3007 East Asian subjects. In the output file of SNP2HLA, imputed classical HLA alleles and HLA protein amino acid positions were defined as binary markers coding the presence or absence of the allele or residue being tested, and each different allele or residue was tested as a biallelic position. Association with AS was then tested using logistic regression function in PLINK [10] by including all allele/residues/SNP conditioning on 10 principal components to control for population stratification effects. We then performed conditional analysis repeatedly in an iterative fashion by adding the dosage of HLA-B*27 allele and other significant alleles/residues/SNPs as covariates until no significant allele/residue/SNP was observed. Only $H L A$ alleles or amino acids with imputation information scores $>0.5$ were considered. All results are presented unadjusted for multiple testing.

\section{Results}

PCA indicated that all study subjects were ethnically East Asian (Supplementary Figure 1). The genomic inflation factor calculated using a set of 1767 negative control SNPs in regions included on Immunochip for studies of reading and writing disabilities, psychosis, and schizophrenia was 1.03 (lambda $(1000)=1.02$ ). No evidence of statistical inflation is seen in the Q-Q plot (Supplementary Figure 2). After quality control and imputation, 15,748 SNPs across the MHC (from 25 to 35 $\mathrm{Mb}$, hg18) were available for analysis in 1482 cases and 1512 controls. Imputed HLA-B allele frequencies amongst controls in the current study were not significantly different from those in previously reported directly genotyped studies $(P>0.05)$, confirming the high accuracy of HLA imputation, particularly at a two-digit resolution [3].

\section{HLA-B associations}

The strongest SNP association with AS observed was a missense variant of $H L A-B, \quad$ rs1071652 (SNPB $* 31432180 \_C G, \quad$ odds ratio $(\mathrm{OR})=180, \quad P=4.45 \times$ $10^{-256}$, Fig. 1). The previously reported East Asian HLA$B^{*} 27$ tagSNP rs13202464 (31452562) [11] was also found significantly associated with AS $(\mathrm{OR}=58.73, P=1.92 \times$ $\left.10^{-211}\right)$. Controlling for rs1071652, residual association is seen with HLA-B $27\left(4.48 \times 10^{-22}\right)$ and SNP rs41553720 (SNP-B*31432843_A, $P=3.87 \times 10^{-31}$ ), indicating that combinations of SNPs are currently required to tag HLA-B*27 in East Asian populations, in contrast to the situation in European descent populations [12].

After SNP imputation in the MHC region, the expected strong association was observed with HLA-B*27 (odds ratio $(\mathrm{OR})=205, P=5.76 \times 10^{-244}$, Table 2).
Controlling for the HLA-B*27 association and studying other HLA-B alleles, risk association is seen with HLA$B * 40$ at suggestive level $\left(O R=1.65, P=2.54 \times 10^{-4}\right)$. Controlling for both HLA-B*27 and HLA-B*40, no association was observed in the 2-digit HLA-B allele with MAF $>1 \%$ (only rare alleles HLA-B*53 and HLA-B*38 were associated at suggestive level; $P$ values were $2.9 \times$ $10^{-4}$ and $3.3 \times 10^{-4}$, respectively).

At the amino acid level, the strongest association seen in the uncontrolled analysis was with histidine at position 114 in HLA-B $\left(P=7.24 \times 10^{-241}\right)$, followed by multiple HLA-B amino acids including lysine at $70(P=$ $\left.1.49 \times 10^{-237}\right)$ and asparagine $97\left(P=2.51 \times 10^{-237}\right)$ (Table 3). Asparagine 97 and histidine 114 were previously reported to be the main amino acid determining HLA-B associations with AS in European descent and Korean populations, respectively $[1,2]$.

Conditional analyses for these individual amino acids and their combinations reveal that only the association of histidine 114 can be attenuated by conditioning on asparagine 97 (Table 4). No other individual amino acid explains the association of the other amino acids. For HLA-B alleles, both combinations of lysine $70+$ asparagine 97 and lysine $70+$ histidine 114, but not asparagine 97 + histidine 114, controlled for association with any other 2-digit HLA-B allele $(P>0.0017$, correcting for 30 2-digit HLA-B alleles tested). Controlling for all of lysine 70 , asparagine 97 , and histidine 114, the strongest HLA amino acid association remains with several positions including HLA-B position 97 (serine, found on HLA-B*7, "8, "15, "2707, *40, "41, "48; OR = 2.14, $P=2.14 \times 10^{-5}$ ).

Controlling for $H L A-B * 27$ alone or in combination with $H L A-B * 40$ did not fully control for the association of asparagine 97 , lysine 70 , or histidine $114\left(P<5 \times 10^{-8}\right.$ for both analyses, Table 3).

\section{Non-HLA-B susceptibility loci in the MHC}

Considering HLA alleles other than HLA-B, several HLA-A, HLA-C, and HLA class II alleles showed significant associations (Table 2). Controlling for the HLA$B * 27$ association, independent risk association was confirmed with $H L A-C^{*} 15\left(\mathrm{OR}=2.13, P=9.30 \times 10^{-19}\right)$ and $H L A-C^{*} 1502\left(\mathrm{OR}=7.62, P=6.78 \times 10^{-23}\right)$, both associations at the amino acid level tagged by a leucine 116 in HLA-C $\left(P=6.61 \times 10^{-21}\right)$ located in the HLA-C epitopebinding groove. Stepwise conditional analyses on both $H L A-B * 27$ and $H L A-C * 15$ demonstrated significant associations with $H L A-D Q B 1 * 04\left(\mathrm{OR}=2.13, P=7.91 \times 10^{-5}\right)$ after correcting for multiple comparisons (499 signals across MHC, as defined by regions with $\mathrm{LD} r^{2}<0.2$, Bonferroni correction threshold $\left.=10^{-4}\right)$. Conditioning on both $H L A-B * 27$ and $H L A-B * 40$, the association was confirmed with $H L A-C^{*} 15\left(\mathrm{OR}=4.97, P=9.88 \times 10^{-17}\right)$ and HLA-DQB1*04 (OR $\left.=2.42, P=1.86 \times 10^{-6}\right)$. 

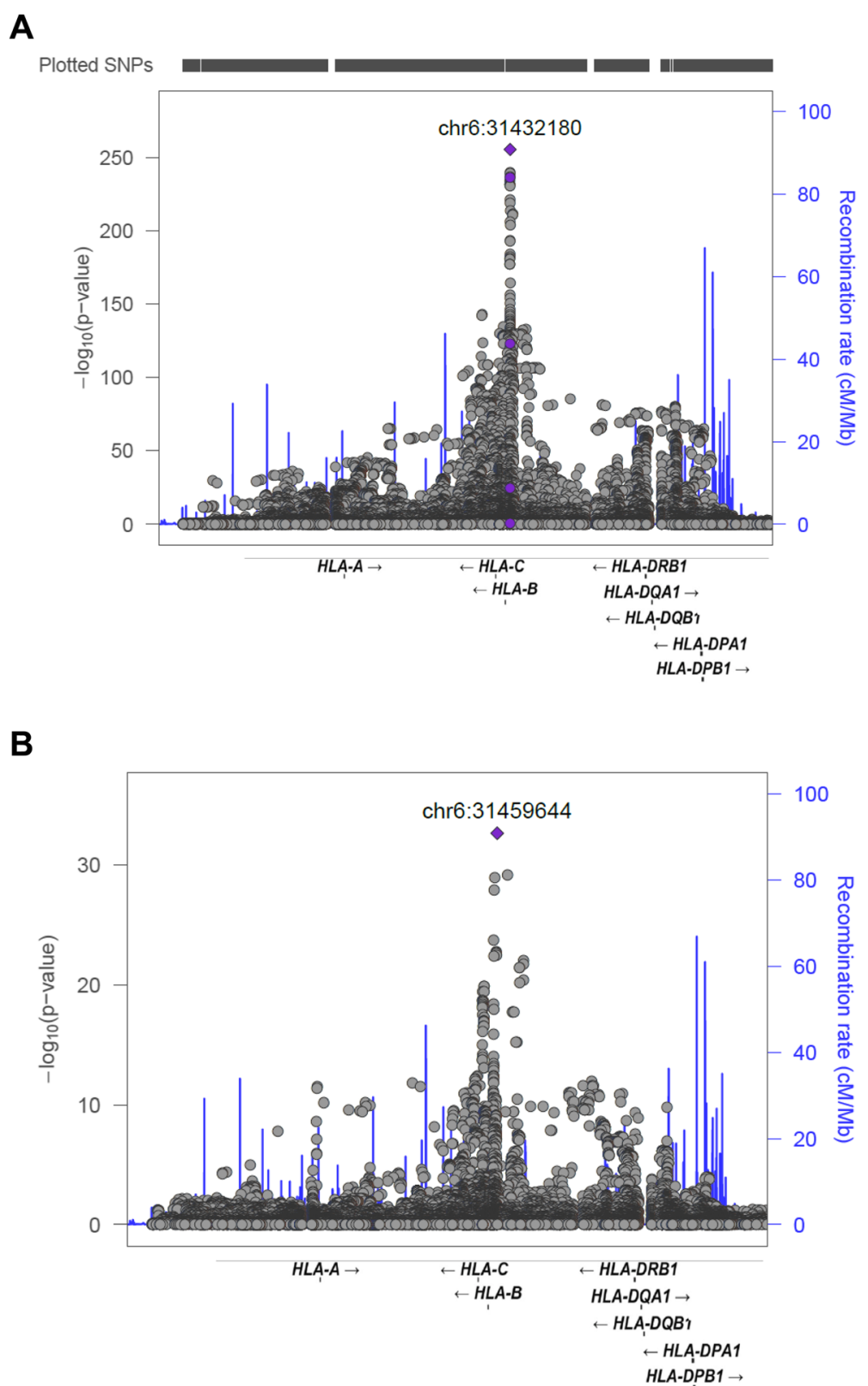

Fig. 1 AS susceptibility associations in the MHC region. Association plots for the extended major histocompatibility complex region. Significance levels of each marker SNPs were calculated by using logistic regression of imputed dosage files and plotted according to chromosomal locations (based on hg19). a Top association was identified at HLA-B27. b There were residual signals with SNPs near HLA-A, HLA-B, HLA-C, and HLA class II alleles conditioning on the HLA-B*27

\section{ERAP1 variants in association with AS}

The key ERAP1 variant associated with AS is rs30187 (ccc-5-96150086-T-C, chr5:96150086[hg18], encoding K528R) [4, 13] (Table 5). It has previously been observed in European populations that the association with the variant rs30187 in the ERAP1 locus is restricted to HLA$B * 27$-positive subjects, or HLA-B*40-positive, HLA-B27negative subjects, consistent with epistatic interactions. Here, we investigated the possibility of interaction between the HLA-B*27 and HLA-B*40 alleles and the previously reported tag SNP of ERAP1 locus (rs30187) [4]. When testing for interaction with the $H L A-B * 27$ alleles, we found that rs30187-A risk allele increased the risk of disease in the strata where $H L A-B * 27$ was present $\left(\mathrm{OR}=1.29 ; P=2.71 \times 10^{-6}\right)($ Table 5$)$, but no association was seen in HLA-B27-negative cases $(\mathrm{OR}=1.06, P=$ $0.61)$. No evidence of interaction was observed between rs30187 and the $H L A-B * 40$ allele, although the power to identify this was low as the number of HLA-B27negative cases was low. 
Table 2 Association of HLA alleles with susceptibility to ankylosing spondylitis ( $P<0.05$ in conditional analysis on HLA-B27)

\begin{tabular}{|c|c|c|c|c|c|}
\hline SNP & $\mathrm{FRQ}$ & OR & $P$ & P_con_B27 & P_con_B27_B40 \\
\hline \multicolumn{6}{|l|}{ 2-digit HLA alleles } \\
\hline HLA-C $C^{*} 15$ & 0.04 & 1.01 & 0.97 & $9.30 \times 10^{-19}$ & $9.88 \times 10^{-17}$ \\
\hline HLA-DQB1*04 & 0.07 & 0.96 & 0.68 & $5.98 \times 10^{-7}$ & $1.86 \times 10^{-6}$ \\
\hline HLA-DRB $1 * 13$ & 0.07 & 0.37 & $6.28 \times 10^{-15}$ & $2.53 \times 10^{-4}$ & $7.85 \times 10^{-4}$ \\
\hline$H L A-B^{*} 40$ & 0.12 & 0.58 & $2.63 \times 10^{-11}$ & $2.54 \times 10^{-4}$ & NA \\
\hline$H L A-C^{*} 03$ & 0.20 & 0.43 & $9.19 \times 10^{-33}$ & $6.08 \times 10^{-4}$ & $9.92 \times 10^{-5}$ \\
\hline HLA-DQB1*06 & 0.22 & 0.52 & $1.50 \times 10^{-20}$ & $6.32 \times 10^{-4}$ & $1.25 \times 10^{-3}$ \\
\hline$H L A-B * 53$ & 0.00 & 0.62 & 0.46 & $7.33 \times 10^{-4}$ & $2.87 \times 10^{-4}$ \\
\hline$H L A-B^{*} 38$ & 0.01 & 2.10 & 0.041 & $9.55 \times 10^{-4}$ & $3.35 \times 10^{-4}$ \\
\hline HLA-C*05 & 0.01 & 0.25 & $2.75 \times 10^{-3}$ & $1.77 \times 10^{-3}$ & $2.68 \times 10^{-3}$ \\
\hline$H L A-B^{*} 15$ & 0.10 & 0.40 & $1.13 \times 10^{-21}$ & 0.021 & 0.082 \\
\hline$H L A-D R B 1 * 04$ & 0.15 & 1.09 & 0.26 & 0.028 & 0.032 \\
\hline$H L A-D P B 1 * 45$ & 0.00 & 0.98 & 0.97 & 0.029 & 0.025 \\
\hline$H L A-D Q A 1 * 06$ & 0.06 & 4.09 & $3.05 \times 10^{-17}$ & 0.048 & 0.035 \\
\hline \multicolumn{6}{|l|}{ 4-digit HLA alleles } \\
\hline HLA-C ${ }^{*} 1502$ & 0.03 & 1.15 & 0.37 & $6.78 \times 10^{-23}$ & $4.10 \times 10^{-20}$ \\
\hline$H L A-B^{*} 4002$ & 0.03 & 0.79 & 0.16 & $9.55 \times 10^{-12}$ & $6.54 \times 10^{-8}$ \\
\hline HLA-DRB 1*0405 & 0.06 & 0.93 & 0.51 & $2.30 \times 10^{-7}$ & $1.06 \times 10^{-6}$ \\
\hline HLA-DQB1 ${ }^{* 0401}$ & 0.05 & 0.90 & 0.45 & $5.05 \times 10^{-6}$ & $1.54 \times 10^{-5}$ \\
\hline HLA-B*4005 & 0.00 & 1.72 & 0.55 & $4.21 \times 10^{-4}$ & $2.09 \times 10^{-3}$ \\
\hline$H L A-B * 5301$ & 0.00 & 0.62 & 0.46 & $7.20 \times 10^{-4}$ & $2.82 \times 10^{-4}$ \\
\hline HLA-DRB ${ }^{*} 1302$ & 0.05 & 0.37 & $5.63 \times 10^{-13}$ & $1.13 \times 10^{-3}$ & $2.88 \times 10^{-3}$ \\
\hline HLA-B*3802 & 0.01 & 2.29 & 0.036 & $2.16 \times 10^{-3}$ & $1.01 \times 10^{-3}$ \\
\hline HLA-C*0501 & 0.00 & 0.38 & 0.054 & $3.98 \times 10^{-3}$ & $5.60 \times 10^{-3}$ \\
\hline HLA-DQA1*0102 & 0.27 & 0.36 & $3.45 \times 10^{-30}$ & $4.68 \times 10^{-3}$ & 0.013 \\
\hline$H L A-B^{*} 4446$ & 0.03 & 0.21 & $5.69 \times 10^{-14}$ & $6.15 \times 10^{-3}$ & 0.012 \\
\hline HLA-C*0304 & 0.08 & 0.53 & $1.72 \times 10^{-9}$ & 0.012 & $6.95 \times 10^{-5}$ \\
\hline HLA-DRB1*1501 & 0.08 & 0.56 & $5.36 \times 10^{-8}$ & 0.022 & 0.024 \\
\hline$H L A-B^{*} 4402$ & 0.00 & 0.30 & 0.13 & 0.027 & 0.033 \\
\hline$H L A-D P B 1 * 4501$ & 0.00 & 0.97 & 0.96 & 0.029 & 0.024 \\
\hline$H L A-D Q B 1^{*} 0603$ & 0.01 & 0.41 & $2.55 \times 10^{-3}$ & 0.036 & 0.055 \\
\hline$H L A-B^{*} 1501$ & 0.04 & 0.24 & $2.94 \times 10^{-15}$ & 0.047 & 0.11 \\
\hline HLA-DQA1*0601 & 0.06 & 4.09 & $3.04 \times 10^{-17}$ & 0.048 & 0.035 \\
\hline HLA-B*5502 & 0.02 & 0.28 & $9.64 \times 10^{-6}$ & 0.050 & 0.10 \\
\hline
\end{tabular}

$O R$ odds ratio in unconditional analysis, $F R Q$ allele frequency in controls, $P P$ value in unconditional analysis, $P_{-}$con_B27 $P$ value in conditional analysis controlling HLA-B*27, P_con_B27_B40 P value in conditional analysis controlling HLA-B*27 and HLA-B*40

\section{Discussion}

This study confirms that in East Asians, the primary MHC associations with $\mathrm{AS}$ are with $H L A-B * 27$ and $H L A-B * 40$, and confirms the risk association of $H L A$ $C * 1502$ with the disease. The association of $H L A-B * 40$ with AS has been convincingly demonstrated now in both European descent $[1,14-16]$ and East Asian studies $[3,17]$, using both direct genotyping- and imputationbased methods. $H L A-B * 4001$ has also been shown to be associated with $\operatorname{IgA}$ nephropathy $(\mathrm{OR}=1.34, P=5.64 \times$ $10^{-7}$ ) [18], a known though uncommon association of AS. The functional mechanism of association of this allele has been little studied. It does not share the lysine 70, asparagine 97, or histidine 114 residues found in most $H L A-B * 27$ alleles. As with $H L A-B * 27$, it is known to interact with AS-associated ERAP1 variants to cause AS, suggesting that it is likely to operate by the same mechanism. Further studies to compare its properties 
Table 3 Association of amino acid residues in HLA-B with susceptibility to ankylosing spondylitis $\left(P<10^{-100}\right.$ in unconditional analysis)

\begin{tabular}{|c|c|c|c|c|c|c|}
\hline Position & AA & FRQ & OR & $P$ & P_con_B27 & P_con_B27_B40 \\
\hline 114 & $\mathrm{H}$ & 0.24 & 232.78 & $7.24 \times 10^{-241}$ & $1.86 \times 10^{-8}$ & $1.18 \times 10^{-8}$ \\
\hline 70 & K & 0.25 & 312.90 & $1.49 \times 10^{-237}$ & $1.22 \times 10^{-40}$ & $1.35 \times 10^{-38}$ \\
\hline 97 & N & 0.24 & 255.79 & $2.51 \times 10^{-237}$ & $1.08 \times 10^{-13}$ & $3.49 \times 10^{-13}$ \\
\hline 67 & C & 0.30 & 62.52 & $8.05 \times 10^{-232}$ & $6.91 \times 10^{-17}$ & $6.89 \times 10^{-18}$ \\
\hline 116 & D & 0.29 & 57.93 & $2.38 \times 10^{-221}$ & 0.15 & 0.061 \\
\hline 80 & $\mathrm{~T}$ & 0.31 & 42.93 & $1.99 \times 10^{-220}$ & $2.82 \times 10^{-20}$ & $1.27 \times 10^{-21}$ \\
\hline 113 & Y & 0.32 & 25.09 & $5.03 \times 10^{-194}$ & 0.33 & 0.12 \\
\hline 9 & $\mathrm{H}$ & 0.39 & 16.96 & $2.20 \times 10^{-154}$ & $1.00 \times 10^{-11}$ & $3.64 \times 10^{-14}$ \\
\hline 45 & E & 0.39 & 14.84 & $5.35 \times 10^{-153}$ & $2.41 \times 10^{-9}$ & $3.46 \times 10^{-11}$ \\
\hline 9 & Y & 0.39 & 16.08 & $1.75 \times 10^{-151}$ & $3.24 \times 10^{-11}$ & $2.68 \times 10^{-12}$ \\
\hline 69 & A & 0.40 & 11.19 & $1.81 \times 10^{-146}$ & $8.20 \times 10^{-7}$ & $1.13 \times 10^{-8}$ \\
\hline 11 & S & 0.41 & 13.63 & $4.25 \times 10^{-145}$ & $8.62 \times 10^{-14}$ & $1.66 \times 10^{-11}$ \\
\hline 83 & $\mathrm{R}$ & 0.42 & 13.77 & $8.00 \times 10^{-145}$ & $1.76 \times 10^{-14}$ & $7.46 \times 10^{-18}$ \\
\hline 12 & v & 0.41 & 12.47 & $9.64 \times 10^{-143}$ & $6.01 \times 10^{-13}$ & $9.44 \times 10^{-11}$ \\
\hline 82 & L & 0.44 & 10.47 & $2.64 \times 10^{-140}$ & $8.24 \times 10^{-12}$ & $3.80 \times 10^{-15}$ \\
\hline 32 & $\mathrm{~L}$ & 0.41 & 11.00 & $7.76 \times 10^{-137}$ & $3.06 \times 10^{-7}$ & $1.38 \times 10^{-4}$ \\
\hline 80 & $\mathrm{~N}$ & 0.45 & 8.89 & $3.98 \times 10^{-135}$ & $8.83 \times 10^{-13}$ & $1.49 \times 10^{-16}$ \\
\hline 24 & $\mathrm{~T}$ & 0.44 & 9.12 & $2.58 \times 10^{-134}$ & $3.08 \times 10^{-9}$ & $8.53 \times 10^{-7}$ \\
\hline 77 & D & 0.14 & 27.62 & $5.68 \times 10^{-128}$ & 0.39 & 0.39 \\
\hline 74 & D & 0.43 & 7.41 & $6.83 \times 10^{-126}$ & $7.02 \times 10^{-3}$ & $2.97 \times 10^{-4}$ \\
\hline 163 & $\mathrm{E}$ & 0.46 & 6.58 & $1.53 \times 10^{-121}$ & $2.06 \times 10^{-3}$ & 0.38 \\
\hline 69 & $\mathrm{~T}$ & 0.46 & 5.72 & $4.97 \times 10^{-117}$ & $5.01 \times 10^{-4}$ & $5.62 \times 10^{-6}$ \\
\hline-16 & $\mathrm{~L}$ & 0.48 & 5.77 & $4.58 \times 10^{-116}$ & $1.76 \times 10^{-6}$ & $1.28 \times 10^{-5}$ \\
\hline-16 & V & 0.48 & 5.69 & $2.93 \times 10^{-115}$ & $1.66 \times 10^{-6}$ & $1.17 \times 10^{-5}$ \\
\hline 71 & A & 0.47 & 5.49 & $5.24 \times 10^{-114}$ & $1.94 \times 10^{-3}$ & $3.15 \times 10^{-5}$ \\
\hline 70 & N & 0.47 & 5.48 & $5.55 \times 10^{-114}$ & $1.96 \times 10^{-3}$ & $3.19 \times 10^{-5}$ \\
\hline 97 & $\mathrm{R}$ & 0.54 & 5.13 & $9.25 \times 10^{-109}$ & $7.34 \times 10^{-7}$ & $2.13 \times 10^{-6}$ \\
\hline 24 & A & 0.43 & 0.23 & $6.90 \times 10^{-103}$ & $3.58 \times 10^{-8}$ & $1.14 \times 10^{-5}$ \\
\hline
\end{tabular}

$O R$ odds ratio in unconditional analysis, $P P$ value in unconditional analysis, $P$ con $B 27 P$ value in conditional analysis controlling $H L A-B * 27$,

$P \_$con_B27_B40 $P$ value in conditional analysis controlling HLA-B*27 and HLA-B*40. NS $=P>0.05$

with HLA-B27, such as its peptide-binding characteristics, folding rate, and whether it forms homodimers, are indicated to investigate its association further.

No protective association was seen with $H L A-B * 07$ as has previously been reported in East Asians [3] and European descent cohorts $[1,15]$, although the allele frequency was very low and the study may not have had adequate power to detect any association with the allele (frequency $=0.024)$.

The study indicates that in East Asians, the key amino acid drivers of the HLA-B associations in AS are amino acid positions 70 and 97 . These remain AS-associated controlling for any other HLA-B amino acid. HLA-B position 97 was previously shown in European descent cohorts to be the key amino acid association in the broad ethnicity, whereas in a Korean study, the association of histidine 114 could not be distinguished from associations with lysine 70 and asparagine 97 [2]. The difference in these findings may be explained by three key factors, sample size, ethnicity, and the reference haplotype dataset. Cortes et al.'s study of European descent subjects involved 9069 AS cases and 13578 controls, over seven times as many subjects as involved in the current study (1637 cases, 1589 controls) and nearly six times the number involved in the previous Korean study (654 cases, 3166 controls). Therefore, the European descent study had greater power, potentially explaining the absence of signal in the East Asian cohorts for some of the HLA-B allele and the HLA class II associations seen in the European dataset. The European descent study also has greater power in conditional analyses, potentially explaining the differences in results regarding the role of lysine 70 , which remains positively associated with AS after conditioning on asparagine 97 in the current study, but not in the European descent dataset. The different studies have also used different reference haplotype datasets, potentially affecting the accuracy of the imputation data. Ethnic differences could also play a role through differences in $H L A-B * 27$ subtypes or other HLA-B allele frequencies, particularly comparing the European descent and East Asian cohorts.

Both HLA-B amino acid residues 70 and 97 are found within the $\mathrm{B}$ pocket of the HLA-B peptide-binding groove. However, it has been noted that position 70 is tightly coupled with positions 67 and 97 and that position 70 hardly changes the peptide-binding repertoire, suggesting that position 70 is "hitch-hiking" along with positions 67 and 97 in their ability to change the peptide-binding repertoire [19]. Our study and the previous HLA amino acid imputation studies suggest that other amino acid positions in addition to 70 (like position 97 and 114) are also involved in HLA-B risk attribution. The association of these amino acids independent of other amino acids found in the HLAB27 B pocket, and having controlled for HLA-B27, indicates that their effect on disease risk is partially independent of HLA-B27.

Although the HLA allele frequencies imputed in controls in this study closely match those reported by direct genotyping studies in Han Chinese [3], the accuracy of imputation in such studies is very dependent on the ethnic matching of the imputed and reference datasets. Whilst the Han-MHC reference dataset used here is of large size $(n=9689)$, the number of East Asian in 1000 Genomes Phase $3(n=524)$, which we used in the Michigan Imputation Server, is far smaller than the European 
Table 4 Conditional analysis $P$ values of HLA-B amino acid residues. Significance for association of lysine 70 (70K), asparagine 97 $(97 \mathrm{~N})$, and histidine $114(114 \mathrm{H})$ is given in columns, either in unconditional analysis or conditioning on specific amino acid positions (where no letter is given after the HLA-B amino acid position number) or for specific amino acids (where a letter is given after the $\mathrm{HLA}-\mathrm{B}$ amino acid position number), either individually or in combinations. NS $=P>0.05$

\begin{tabular}{lllll}
\hline & $70 \mathrm{~K}$ & $97 \mathrm{~N}$ & $114 \mathrm{H}$ & $\mathrm{HLA}-\mathrm{B}^{*} 27$ \\
\hline Unconditional & $1.49 \times 10^{-237}$ & $2.51 \times 10^{-237}$ & $7.24 \times 10^{-241}$ & $5.76 \times 10^{-244}$ \\
70 & - & $1.10 \times 10^{-6}$ & $1.76 \times 10^{-6}$ & $0.38 \times 10^{-7}$ \\
97 & $1.04 \times 10^{-28}$ & - & - & 0.13 \\
114 & $4.08 \times 10^{-37}$ & $2.70 \times 10^{-8}$ & 0.24 & $0.25 \times 10^{-6}$ \\
$70+97$ & - & - & - & 0.11 \\
$70+114$ & - & $2.94 \times 10^{-1}$ & - & 0.20 \\
$97+114$ & $5.81 \times 10^{-28}$ & - & - & 0.066 \\
$70+97+114$ & - & - & $1.04 \times 10^{-6}$ & 0.21 \\
$70 \mathrm{~K}$ & - & $6.15 \times 10^{-7}$ & 0.42 & $4.83 \times 10^{-7}$ \\
$97 \mathrm{~N}$ & $1.11 \times 10^{-31}$ & - & - & 0.020 \\
$114 \mathrm{H}$ & $1.70 \times 10^{-38}$ & $2.02 \times 10^{-8}$ & 0.49 & $2.47 \times 10^{-6}$ \\
$70 \mathrm{~K}+97 \mathrm{~N}$ & - & - & - & 0.20 \\
$70 \mathrm{~K}+114 \mathrm{H}$ & - & 0.30 & - & 0.18 \\
$97 \mathrm{~N}+114 \mathrm{H}$ & $1.27 \times 10^{-31}$ & - & - & 0.32 \\
$70 \mathrm{~K}+97 \mathrm{~N}+114 \mathrm{H}$ & - & - & 0.28 \\
\hline
\end{tabular}

dataset used in Cortes et al. (Type 1 Diabetes Genetics Consortium dataset, $n=5225$ ) [1]. The smaller reference dataset size precluded imputation to four-digit levels and may have affected the accuracy of the imputation of low-frequency alleles in particular. As SNP-based HLA imputation is a highly efficient method enabling largescale HLA association studies, there is a clear need for much larger publicly available HLA imputation reference datasets for Asian populations.

In this study, we have also confirmed the interaction between ERAP1 and $H L A-B * 27$, with association only observed of the key ERAP1 variant, rs30187, only seen in $H L A-B * 27$ -

Table 5 Association analysis of rs30187 in samples positive and negative for $H L A-B^{*} 27$ and $H L A-B^{*} 40$. Odds ratios are given for the rs30187-risk $A$ allele

\begin{tabular}{lll}
\hline Group (case/control) & OR & $P$ \\
\hline All (1482/1512) & 1.26 & $9.00 \times 10^{-6}$ \\
HLA-B27+ cases vs all controls (1323/1512) & 1.29 & $2.71 \times 10^{-6}$ \\
HLA-B27- cases vs all controls (159/1512) & 1.06 & 0.61 \\
HLA-B27+ cases and controls (1323/77) & 1.40 & 0.044 \\
HLA-B27- cases and controls (159/1435) & 1.06 & 0.64 \\
HLA-B27+ cases vs HLA-B27- cases (1323/159) & 1.21 & 0.11 \\
HLA-B27+/HLA-B40+ (207/11) & 1.25 & 0.60 \\
HLA-B27+/HLA-B40- (1116/66) & 1.42 & 0.050 \\
HLA-B27-/HLA-B40- (89/1027) & 1.11 & 0.49 \\
HLA-B27-/HLA-B40+ (70/408) & 1.02 & 0.91 \\
\hline
\end{tabular}

$O R$ odds ratio in unconditional analysis, $P P$ value in unconditional analysis, $S E$ standard error of beta (log-odds) estimate, CHR chromosome positive individuals. This confirms the original finding in Europeans [1] and the previous finding in a case-only analysis of Taiwanese AS patients of different ERAP1 genotypes in $H L A-B * 27$-positive and $H L A-B * 27$-negative cases [20]. We did not see an association of ERAP1 variants in $H L A-B * 27$ negative and $H L A-B * 40$-positive individuals as previously reported [1], although the sample size was not large. The confirmation of the gene-gene interaction in an East Asian population increases the evidence that this is a true-positive interaction and is critical to AS pathogenesis.

\section{Conclusions}

This study confirms that the HLA associations of AS are complex and that multiple non-HLA-B 27 alleles, including both HLA class I and likely HLA class II variants, also contribute to risk and protection from the disease. Further investigation of the mechanisms involved in these associations is likely to assist in determining the pathogenesis of this disease.

\section{Supplementary information}

Supplementary information accompanies this paper at https://doi.org/10. 1186/s13075-020-02148-5.

\section{Additional file 1}

\section{Abbreviations}

AS: Ankylosing spondylitis; HGDP-CEPH: Human Genome Diversity Panel; MHC: Major histocompatibility complex; MAF: Minor allele frequency; OR: Odds ratio; PCs: Principal components; PCA: Principal components analysis; SNP: Single nucleotide polymorphism 


\section{Acknowledgements}

The authors would like to thank Erika de Guzman, Sharon Song, and Lisa Anderson from the Australian Translational Genomics Centre (https:// research.qut.edu.au/translationalgenomicsgroup/atgc/) for their assistance in SNP microarray genotyping.

\section{Authors' contributions}

The study design and case recruitment was performed by GW, THK, KK, SYB, $M A B$, and $H X$. The data analysis was performed by GW, ZL, and AC. The study manuscript was prepared by GW, MAB, and HX. All authors read and approved the final manuscript.

\section{Funding}

MAB was funded by a National Health and Medical Research Council (Australia) Senior Principal Research Fellowship (1024879) and Queensland State Premier's Fellowship for Science. Support for this study was received from a National Health and Medical Research Council (Australia) programme grant (566938) and project grant (569829). This research was funded/supported by the National Institute for Health Research (NIHR) Biomedical Research Centre based at Guy's and St Thomas' NHS Foundation Trust and King's College London and/or the NIHR Clinical Research Facility. The views expressed are those of the authors and not necessarily those of the NHS, the NIHR, or the Department of Health. HX was supported by the National Natural Science Foundation of China (Grant 31821003) and the China Ministry of Science and Technology (Grant 2018AAA0100300).

\section{Availability of data and materials}

Summary data for the datasets used are available at Harvard Dataverse (https://doi.org/10.7910/DVN/NJ7XSO) and on request from the authors.

\section{Ethics approval and consent to participate}

This study protocol was reviewed and approved by the relevant ethics committees of the hospitals and institutions involved. All subjects provided written informed consent.

\section{Consent for publication}

Not applicable.

\section{Competing interests}

The authors declare that they have no competing interests.

\section{Author details}

'Department of Rheumatology and Immunology, Shanghai Changzheng Hospital, Second Military Medical University, Shanghai, China. ${ }^{2}$ University of Queensland Diamantina Institute, University of Queensland, Brisbane, Australia. ${ }^{3}$ Department of Rheumatology, Hanyang University Hospital for Rheumatic Diseases, Seoul, Republic of Korea. ${ }^{4}$ Translational Genomics Group, Institute of Health and Biomedical Innovation, Queensland University of Technology, Translational Research Institute, Brisbane, Australia. ${ }^{5}$ Wellcome Trust Centre for Human Genetics, University of Oxford, Oxford, UK. ${ }^{6}$ Department of Biology, Kyung Hee University, Seoul, Republic of Korea. 7Guy's \& St Thomas' NHS Foundation Trust and King's College London NIHR Biomedical Research Centre, London, England. ${ }^{8}$ Beijing Tsinghua Changgung Hospital, School of Clinical Medicine, Tsinghua University, Beijing 100084, China. ${ }^{9}$ Peking-Tsinghua Center for Life Sciences, Tsinghua University, Beijing, China.

\section{Received: 5 January 2020 Accepted: 6 March 2020}

\section{Published online: 09 April 2020}

\section{References}

1. Cortes A, Pulit SL, Leo PJ, Pointon JJ, Robinson PC, Weisman MH, Ward M, Gensler LS, Zhou X, Garchon HJ, et al. Major histocompatibility complex associations of ankylosing spondylitis are complex and involve further epistasis with ERAP1. Nat Commun. 2015;6:7146.

2. Kim K, Bang SY, Lee S, Lee HS, Shim SC, Kang YM, Suh CH, Sun C, Nath SK, Bae SC, et al. An HLA-C amino-acid variant in addition to HLA-B*27 confers risk for ankylosing spondylitis in the Korean population. Arthritis Res Ther. 2015;17:342.
3. Yi L, Wang J, Guo X, Espitia MG, Chen E, Assassi S, Zou H, Reveille JD, Zhou $X$. Profiling of HLA-B alleles for association studies with ankylosing spondylitis in the Chinese population. Open Rheumatol J. 2013;7:51-4.

4. International Genetics of Ankylosing Spondylitis C, Cortes A, Hadler J, Pointon JP, Robinson PC, Karaderi T, Leo P, Cremin K, Pryce K, Harris J, et al. Identification of multiple risk variants for ankylosing spondylitis through high-density genotyping of immune-related loci. Nat Genet. 2013;45(7):730-8.

5. van der Linden S, Valkenburg HA, Cats A. Evaluation of diagnostic criteria for ankylosing spondylitis. A proposal for modification of the New York criteria. Arthritis Rheum. 1984;27(4):361-8.

6. Cortes A, Brown MA. Promise and pitfalls of the Immunochip. Arthritis Res Ther. 2011;13(1):101.

7. Lopez Herraez D, Bauchet M, Tang K, Theunert C, Pugach I, Li J, Nandineni MR, Gross A, Scholz M, Stoneking M. Genetic variation and recent positive selection in worldwide human populations: evidence from nearly 1 million SNPs. PLoS One. 2009;4(11):e7888.

8. Das S, Forer L, Schonherr S, Sidore C, Locke AE, Kwong A, Vrieze SI, Chew EY, Levy S, McGue M, et al. Next-generation genotype imputation service and methods. Nat Genet. 2016;48(10):1284-7.

9. Zhou F, Cao H, Zuo X, Zhang T, Zhang X, Liu X, Xu R, Chen G, Zhang Y, Zheng $X$, et al. Deep sequencing of the MHC region in the Chinese population contributes to studies of complex disease. Nat Genet. 2016;48(7): 740-6.

10. Purcell $S$, Neale B, Todd-Brown K, Thomas L, Ferreira MA, Bender D, Maller J, Sklar P, de Bakker PI, Daly MJ, et al. PLINK: a tool set for whole-genome association and population-based linkage analyses. Am J Hum Genet. 2007; 81(3):559-75.

11. Lin Z, Bei JX, Shen M, Li Q, Liao Z, Zhang Y, Lv Q, Wei Q, Low HQ, Guo YM, et al. A genome-wide association study in Han Chinese identifies new susceptibility loci for ankylosing spondylitis. Nat Genet. 2011:44(1):73-7.

12. Evans DM, Spencer CC, Pointon JJ, Su Z, Harvey D, Kochan G, Oppermann $\mathrm{U}$, Dilthey A, Pirinen M, Stone MA, et al. Interaction between ERAP1 and HLA-B27 in ankylosing spondylitis implicates peptide handling in the mechanism for HLA-B27 in disease susceptibility. Nat Genet. 2011;43(8):761-7.

13. Roberts AR, Appleton LH, Cortes A, Vecellio M, Lau J, Watts L, Brown MA, Wordsworth P. ERAP1 association with ankylosing spondylitis is attributable to common genotypes rather than rare haplotype combinations. Proc Natl Acad Sci U S A. 2017;114(3):558-61.

14. Robinson WP, van der Linden SM, Khan MA, Rentsch HU, Cats A, Russell A, Thomson G. HLA-Bw60 increases susceptibility to ankylosing spondylitis in HLA-B27+ patients. Arthritis Rheum. 1989;32(9):1135-41.

15. Diaz-Pena R, Vidal-Castineira JR, Lopez-Vazquez A, Lopez-Larrea C. HLA-B*40: 01 is associated with ankylosing spondylitis in HLA-B27-positive populations. J Rheumatol. 2016;43(6):1255-6.

16. Brown MA, Pile KD, Kennedy LG, Calin A, Darke C, Bell J, Wordsworth BP, Cornelis F. HLA class I associations of ankylosing spondylitis in the white population in the United Kingdom. Ann Rheum Dis. 1996;55(4):268-70.

17. Wei JC, Tsai WC, Lin HS, Tsai CY, Chou CT. HLA-B60 and B61 are strongly associated with ankylosing spondylitis in HLA-B27-negative Taiwan Chinese patients. Rheumatology (Oxford). 2004;43(7):839-42.

18. Yu XQ, Li M, Zhang H, Low HQ, Wei X, Wang JQ, Sun LD, Sim KS, Li Y, Foo $J \mathrm{~N}$, et al. A genome-wide association study in Han Chinese identifies multiple susceptibility loci for IgA nephropathy. Nat Genet. 2011;44(2):178-82.

19. van Deutekom HW, Kesmir C. Zooming into the binding groove of HLA molecules: which positions and which substitutions change peptide binding most? Immunogenetics. 2015;67(8):425-36.

20. Wang CM, Ho HH, Chang SW, Wu YJ, Lin JC, Chang PY, Wu J, Chen JY. ERAP1 genetic variations associated with HLA-B27 interaction and disease severity of syndesmophytes formation in Taiwanese ankylosing spondylitis. Arthritis Res Ther. 2012;14(3):R125.

\section{Publisher's Note}

Springer Nature remains neutral with regard to jurisdictional claims in published maps and institutional affiliations. 